\title{
AVALIAÇÃO DO ESTADO NUTRICIONAL E QUALIDADE DE VIDA DE IDOSOS PORTADORES DE DIABETES MELLITUS TIPO 2
}

Letícia Cristinne Costa da Silva ${ }^{1}$

Luciane Marta Neiva de Oliveira²

resumo

A pesquisa teve como objetivo analisar o estado nutricional e qualidade de vida de idosos portadores de diabetes mellitus tipo 2. A população foi composta por 46 voluntários portadores de diabetes tipo 2, com faixa etária entre 60 e 85 anos, membros de uma Associação de Diabéticos localizada em Teresina (Piauí). Os idosos foram submetidos a um formulário sociodemográfico e, em seguida, foi realizada a avaliação do estado nutricional através de uma miniavaliação nutricional e dados antropométricos. Por fim, avaliou-se a qualidade de vida através do questionário Short Form-36. De acordo com as variáveis sociodemográficas, constatou-se que a maior parte dos participantes são do sexo feminino (69,6\%), com idade entre

\footnotetext{
1 Graduada em Nutrição. Pós-graduanda em Nutrição Clínica e Funcional no Centro Universitário Santo Agostinho (UNIFSA). Nutricionista Clínica. E-mail: leticiaccsnutri@gmail.com.

2 Graduada em Farmácia e Fisioterapia. Mestre em Saúde Pública pela Universidade Americana, Assunção, Paraguai. Coordenadora do Curso tecnológico em Estética e Cosmética e docente nos cursos de Fisioterapia, Farmácia e Nutrição do Centro Universitário Santo Agostinho. E-mail: lucianemarta@hotmail.com.
} 
60 e 65 anos (54,3\%), casados (63\%), com ensino médio completo $(54,3 \%)$ e renda de 1 (39,5\%) e 2-3 salários mínimos (39,1\%). Na avaliação do estado nutricional, de acordo com o índice de massa corporal, predominou o sobrepeso em 52,2\%; Pela avaliação da dobra cutânea tricipital, constatou-se obesidade em 45,7\% dos participantes; conforme a circunferência da cintura verificou-se risco muito elevado para o desenvolvimento de doenças cardiovasculares em 69,65\% dos sujeitos pesquisados; constatou-se ainda que 88,43\% dos idosos não apresentaram risco nutricional quando avaliados por meio da miniavaliação nutricional. Quanto à análise de qualidade de vida, as menores médias (escores) foram encontradas no domínio estado geral da saúde $(59,67)$ e dor $(65,63)$. Por conseguinte, os idosos portadores de diabetes tipo 2 avaliados possuem alterações no estado nutricional, entretanto apresentam boa qualidade vida.

\section{palavras-chave}

Diabetes Mellitus. Estado Nutricional. Qualidade de Vida. Idosos.

\section{Introdução}

O diabetes mellitus (DM) é um grupo heterogêneo de distúrbios metabólicos que ocasionam hiperglicemia em decorrência de defeitos na ação da insulina, na secreção de insulina ou em ambas. O DM atualmente configura-se como uma epidemia mundial, devido aos altos índices epidemiológicos. O envelhecimento da população, obesidade, sedentarismo e a crescente urbanização são alguns fatores responsáveis no desenvolvimento da doença que é considerado um problema de saúde pública, devido a elevada prevalência e mortalidade, em decorrência das complicações (SOCIEDADE BRASILEIRA DE DIABETES, 2016; PALMEIRA; PINTO, 2015).

Conforme Deus e Conceição (2012), entre os tipos de DM, destaca-se o tipo 2, que representa cerca de 90\% dos casos. Segundo Souza e Araújo (2015), há aproximadamente 300 milhões de pessoas diabéticas tipo 2 no mundo e $20 \%$ destas possuem idade entre 65 e 76 anos. No Brasil, a prevalência é de $7,6 \%$ da população, entretanto, metade das pessoas acometidas pela doença desconhece o diagnóstico. Portanto, é relevante conhecer o portador desta patologia a fim de identificar intervenções eficazes de prevenção, promoção e controle da doença e dos seus agravos nos serviços de saúde. 
Umbelino, Serrano e Cruz (2008) mencionam que a avaliação nutricional dos diabéticos é um importante instrumento para diagnóstico do estado nutricional e identificação dos fatores de riscos e complicações associadas ao DM, pois, colaboram para aplicação efetiva da dietoterapia, a fim de melhorar a saúde, a sobrevida, e qualidade de vida (QV) dos pacientes, além da prevenção e controle dos agravos decorrentes da doença.

De acordo com Coqueiro et al. (2009), a avaliação do estado nutricional pode ser realizada através de dados antropométricos (peso, estatura, perímetros e dobras cutâneas), parâmetros bioquímicos, exame físico, inquéritos alimentares e composição corporal. A antropometria é a mais utilizada por ser segura, de fácil execução e baixo custo.

Esta patologia, independentemente da etiologia, gênero, faixa etária, escolaridade, nível socioeconômico, pode causar impacto negativo que compromete a QV. Uma vez que, o diagnóstico ocasiona mudanças na rotina do indivíduo, devido à adesão ao uso de medicamentos (orais ou de invasivos) e novos hábitos alimentares, podendo elevar os níveis de estresse do portador, alterando seu estado psicológico, provocando alterações na QV, diferentes aspectos, como debilidade do estado físico, prejuízo da capacidade funcional, dor em membros inferiores, falta de vitalidade, dificuldades no relacionamento social, instabilidade emocional, entre outros (FARIA et al., 2013; FENG; ASTELL-BUR, 2017; ZULIAN et al., 2013).

A avaliação da QV deve ser realizada com questionários padronizados, pois estes possuem questões subjetivas em dados objetivos, que podem ser quantificados e analisados. Os resultados são importantes para verificar o impacto das intervenções em saúde na QV dos pacientes (DIAZ et al., 2016). De acordo com o exposto, a pesquisa teve como objetivo analisar o estado nutricional e qualidade de vida de idosos portadores de diabetes mellitus tipo 2.

\section{Métodos}

Trata-se de um estudo transversal de natureza quantitativa, elaborado a partir do Trabalho de Conclusão de Curso: Avaliação do estado nutricional e qualidade de vida de idosos portadores de diabetes tipo 2, apresentado a Coordenação de Nutrição do Centro Universitário Santo Agostinho (UNIFSA). A pesquisa foi realizada em uma Associação de Diabéticos, localizada em Teresina, Piauí, no período de agosto a novembro de 2017, com os adscritos desta. Os critérios de inclusão propostos foram: idosos de ambos os gêneros com idade de 60 a 85 anos, portadores de diabetes tipo 2, com cadastrado na 
associação e os critérios de exclusão: portadores de diabetes mellitus tipo 1, cadastro desatualizado no sistema da associação, idosos que não frequentavam as reuniões e presença de déficit cognitivo. A Associação tinha 191 associados cadastrados, desta forma, após os critérios de inclusão e exclusão, 46 idosos foram selecionados para participar da pesquisa.

Os princípios éticos foram seguidos em todas as fases do estudo, em consonância com o que preconiza a Resolução no 466/2012. A pesquisa foi submetida ao Comitê de Ética em Pesquisa e aprovada sob o número de parecer: 2.095.171. O estudo foi iniciado somente após a aprovação. Os indivíduos foram convidados a participarem voluntariamente do estudo, orientados acerca do anonimato na pesquisa e assinaram o Termo de Consentimento Livre e Esclarecido (TCLE).

Toda a coleta dos dados e os procedimentos foram realizados na Associação de Diabéticos no período vespertino com horários de início e término pré-definidos. Os participantes foram entrevistados pelas pesquisadoras, primeiramente responderam a um questionário sociodemográfico com questões fechadas em forma de entrevista contendo informações sobre as seguintes variáveis: idade, sexo, grau de instrução, estado civil, renda. Em seguida foi avaliado o estado nutricional utilizando-se o índice de massa corporal (IMC), perímetro da cintura, perímetro da panturrilha, perímetro do braço, dobra cutânea tricipital e perímetro muscular do braço e a Miniavaliação Nutricional (MAN).

Para calcular o IMC, foi realizado a aferição da altura e do peso do participante. Para a aferição da altura foi utilizado a fita métrica que esteja estável sob uma superfície lisa. O participante estava descalço e com a cabeça livre de adereços, de costas para superfície lisa. Em seguida se manteve na posição ortostática. Na aferição do peso, o participante estava posicionado no centro da balança, descalço, ereto, com os pés juntos e braços estendidos ao longo do corpo, ficando parado para a leitura do resultado. O IMC foi calculado pela divisão do peso, em quilos, pela estatura, em metros, elevada ao quadrado. $\mathrm{O}$ resultado é classificado com os valores de referência propostos por Lipschitz (1994) para idosos, sugerido pelo Ministério da Saúde: Baixo peso $\left(<22 \mathrm{~kg} / \mathrm{m}^{2}\right)$, Eutrofia $\left(>22 \mathrm{e}<27 \mathrm{~kg} / \mathrm{m}^{2}\right)$, Sobrepeso $\left(>27 \mathrm{~kg} / \mathrm{m}^{2}\right)$.

O perímetro da cintura (PC) foi avaliado utilizando uma fita métrica flexível, realizando a medida com o participante em pé, no ponto médio entre a última costela e a crista ilíaca, no momento expiração. Os pontos de corte adotados para classificação da PC foram: $>80 \mathrm{~cm}$, para mulheres e $>94 \mathrm{~cm}$, para homens, classificados como risco elevado para doenças cardiovasculares e considerado risco muito elevado $\mathrm{PC}>88 \mathrm{~cm}$ para mulheres e $>102 \mathrm{~cm}$ para homens conforme a National Institutes of Health (NIH) (2011). 
O perímetro da panturrilha (PP) foi aferido na maior proeminência da musculatura da panturrilha, o ponto de corte adotado para classificação de depleção muscular foram valores inferiores a $31 \mathrm{~cm}$. O perímetro do braço (PB) foi medida com o braço do participante relaxado ao longo do corpo, com a aferição no ponto médio entre o processo acromial da escápula e olecrano da ulna. A medida foi avaliada pelo percentual da adequação: adequação do perímetro do braço $(\mathrm{PB})=[\mathrm{PB}$ obtida $[\mathrm{cm}] /$ percentil 50 da $\mathrm{PB}$ para a idade. Após a adequação, classificou-se a PB através dos valores de referência de Blackburn \& Thornton (1979): obesidade (>120 \%), sobrepeso (>120 a 110\%), eutrofia (110 a 90\%), depleção discreta (90 a 80\%), depleção moderada (80 a $60 \%$ ) e depleção grave ( $<60 \%)$.

A dobra cutânea tricipital (DCT) foi aferida na face posterior do braço direito, paralelamente ao eixo longitudinal, no ponto que compreende a distância média entre o acrômio e o processo do olecrano da ulna. Adequação da DCT = [DCT obtida [cm] / percentil 50 da DCT para a idade e gênero] x 100. Após a adequação, classificou-se a DCT através dos valores de referência de Blackburn e Thornton (1979): obesidade ( $>120 \%)$, sobrepeso (>120 a 110\%), eutrofia (110 a $90 \%$ ), desnutrição leve (90 a 80\%), desnutrição moderada ( 80 a 60\%) e desnutrição grave $(<60 \%)$.

O perímetro muscular do braço (PMB) foi obtido por meio da $\mathrm{CB}$ e da dobra cutânea tricipital $(\mathrm{PCT})$ pela seguinte fórmula: $\mathrm{PMB}(\mathrm{cm})=\mathrm{PB}(\mathrm{cm})-$ $[0,314 \times \mathrm{DCT}]$. Adequação da $\mathrm{PMB}=[\mathrm{PMB}$ obtida $[\mathrm{cm}] /$ percentil 50 da PMB para a idade] x 100. Após a adequação, classificou-se a PMB através dos valores de referência de Blackburn e Thornton (1979): eutrofia (90\%), desnutrição leve (80 a 90\%), desnutrição moderada (70 a $80 \%$ ) e depleção grave ( $<70 \%)$.

A MAN (GUIGOZ, 2006) é um questionário composto por dezoito perguntas. A soma escores permite uma identificação do estado nutricional além de identificar riscos. $\mathrm{O}$ escore de 12 pontos ou mais considera o idoso como saudável, sendo desnecessária a aplicação de todo o questionário; para aqueles que atingem 11 pontos ou menos, deve ser considerada a possibilidade de desnutrição e, portanto, o questionário deve ser continuado. Com escore total da MAN, foram utilizados os seguintes pontos de corte: para o estado nutricional adequado $\geq 24$; risco de desnutrição entre 17 e 23,5; desnutrição < 17 .

Para avaliar a qualidade de vida foi utilizado o questionário SF-36 (Short-Form Health Survey, 1992) (CICONELLI, 1997). Este realiza a análise através de oito domínios: capacidade funcional, limitação por aspectos físicos, dor, estado geral da saúde, vitalidade, aspectos sociais, aspectos emocionais, saúde mental. É composto por onze perguntas objetivas cuja avaliação dos resultados será feita mediante a atribuição de escores para cada questão, os quais serão transformados 
numa escala de 0 a 100, onde zero correspondem a uma pior qualidade de vida e 100 a uma melhor qualidade de vida.

Os dados foram submetidos à análise descritiva (média, desvio padrão, erro padrão e intervalo de confiança) e tratados em forma de tabelas, utilizando-se a ferramenta Microsoft Excel (Office 2011) e analisados no programa estatístico SPSS 20.0 com nível de confiança de 95\% e erro padrão de 5\%.

\section{Resultados e discussões}

O perfil sociodemográfico dos idosos com diabetes mellitus tipo 2 da presente pesquisa é caracterizado pela predominância do gênero feminino (69,6\%), apresentando idade entre 60 a 65 anos (54,3\%), com renda de um (39,1\%) a dois/três (39,1\%) salários mínimos. Entre os entrevistados, 63\% afirmaram ser casados e $54,3 \%$ concluíram o Ensino Médio.

Na Tabela 1 estão descritos os resultados relacionados às variáveis sociodemográficas dos idosos portadores de diabetes tipo 2 pesquisados.

Tabela 1 - Características da população estudada segundo as variáveis sociodemográficas ( $n=46)$. Teresina, 2017.

\begin{tabular}{|c|c|c|c|c|c|}
\hline \multicolumn{2}{|l|}{ Variável } & \multirow{2}{*}{$\begin{array}{l}\text { Frequência } \\
32 \\
14 \\
46\end{array}$} & \multirow{2}{*}{$\begin{array}{l}\begin{array}{l}\text { Perc. } \\
\text { (\%) }\end{array} \\
69,6 \\
30,4 \\
100,0\end{array}$} & \multirow{2}{*}{$\begin{array}{l}\text { Erro } \\
\text { padrão }\end{array}$} & \multirow{2}{*}{$\begin{array}{l}\begin{array}{l}\text { Intervalo de } \\
\text { confiança } \\
95 \%\end{array} \\
55,5-82,6 \\
17,4-43,5 \\
---\end{array}$} \\
\hline Sexo & $\begin{array}{l}\text { Feminino } \\
\text { Masculino } \\
\text { Total }\end{array}$ & & & & \\
\hline $\begin{array}{l}\text { Idade } \\
\text { (anos) }\end{array}$ & $\begin{array}{l}60-65 \\
66-70 \\
71-75 \\
76-80 \\
81-85 \\
\text { Total }\end{array}$ & $\begin{array}{l}25 \\
11 \\
6 \\
2 \\
2 \\
46\end{array}$ & $\begin{array}{l}54,3 \\
23,9 \\
13,0 \\
4,3 \\
4,3 \\
100,0\end{array}$ & $\begin{array}{l}7,5 \\
6,2 \\
5,0 \\
3,1 \\
3,0 \\
---\end{array}$ & $\begin{array}{l}39,1-69,6 \\
13,0-37,0 \\
4,3-23,9 \\
0-10,9 \\
0-10,9 \\
---\end{array}$ \\
\hline Estado civil & $\begin{array}{l}\text { Solteiro } \\
\text { Casado } \\
\text { Viúvo } \\
\text { Divorciado } \\
\text { Total }\end{array}$ & $\begin{array}{l}8 \\
29 \\
7 \\
2 \\
46\end{array}$ & $\begin{array}{l}17,4 \\
63,0 \\
15,2 \\
4,3 \\
100,0\end{array}$ & $\begin{array}{l}5,6 \\
6,9 \\
5,3 \\
3,0 \\
---\end{array}$ & $\begin{array}{l}6,5-28,3 \\
50,0-76,1 \\
6,5-26,1 \\
0-10,9 \\
---\end{array}$ \\
\hline $\begin{array}{l}\text { Grau de } \\
\text { instrução }\end{array}$ & $\begin{array}{l}\text { Ensino fundamental completo } \\
\text { Ensino médio completo } \\
\text { Ensino superior completo } \\
\text { Total }\end{array}$ & $\begin{array}{l}11 \\
25 \\
10 \\
46\end{array}$ & $\begin{array}{l}23,9 \\
54,3 \\
21,7 \\
100,0\end{array}$ & $\begin{array}{l}5,3 \\
7,4 \\
6,1 \\
---\end{array}$ & $\begin{array}{l}10,9-37,0 \\
39,1-69,6 \\
10,9-34,8\end{array}$ \\
\hline
\end{tabular}




\begin{tabular}{llllll}
\hline \multicolumn{1}{c}{ Variável } & Frequência & $\begin{array}{l}\text { Perc. } \\
\mathbf{( \% )}\end{array}$ & $\begin{array}{l}\text { Erro } \\
\text { padrão }\end{array}$ & $\begin{array}{l}\text { Intervalo de } \\
\text { confiança } \\
\mathbf{9 5 \%}\end{array}$ \\
\hline Renda & 1 salário mínimo & 18 & 39,1 & 7,0 & $23,9-52,2$ \\
individual & 2-3 salários mínimos & 18 & 39,1 & 7,1 & $26,1-54,3$ \\
& 3-4 salários mínimos & 3 & 6,5 & 3,6 & $0-15,2$ \\
& Mais de 4 salários mínimos & 7 & 15,2 & 5,2 & $6,5-26,1$ \\
& Total & 46 & 100,0 & --- & -- \\
\hline
\end{tabular}

Fonte: Dados da pesquisa, 2017.

Segundo Viegas-Pereira, Rodrigues e Machado (2008), a renda influencia fortemente no acesso a serviços de saúde, que, por sua vez, pode influenciar no nível de percepção, procura por atendimento e controle da doença. Conforme os resultados levantados nesta pesquisa, percebeu-se que a maioria dos idosos apresentam renda individual acima de dois salários mínimos, favorecendo um estilo de vida que permite aos idosos maior controle e tratamento do diabetes mellitus.

No estudo de Esteves et al. (2017), realizado em São João do Rio Preto (SP) com idosos diabéticos e hipertensos, a maior frequência foi do sexo feminino (58,06\%), com faixa etária entre 60 a 65 anos (53,23\%), casados (56,45\%) e renda de 1 salário mínimo (66,13\%), corroborando com a presente pesquisa. Entretanto, a maioria dos participantes concluíram apenas o Ensino Fundamental $(87,10 \%)$, antagonizando com os resultados deste estudo.

Conforme Oliveira et al. (2017), a escolaridade é um aspecto importante relacionado à qualidade de vida, pois a baixa escolaridade implica no acesso à educação em saúde, consequentemente, a adoção de hábitos saudáveis, além de prejudicar o entendimento das orientações realizadas por profissionais de saúde. De acordo com Pereira, Spyrides e Andrade (2016), as condições socioeconômicas interferem na qualidade de vida na velhice, uma vez que idosos com maior renda e maior nível de escolaridade possuem mais acesso aos alimentos, realizando melhores escolhas dietéticas, evitando, assim, distúrbios nutricionais.

O perfil da população estudada foi semelhante ao estudo de Santos et al. (2017), realizado em Porteiras (CE). Dos 30 idosos diabéticos avaliados, 76,7\% eram mulheres, fato associado à maior procura das mulheres por serviços de saúde. Na pesquisa realizada por Tavares e Rodrigues (2008), encontraram-se dados equivalentes: dos 153 indivíduos da terceira idade avaliados, 73,5\% eram do sexo feminino. Observou-se, no presente estudo e na literatura, prevalência do gênero feminino. Este dado é associado à maior procura por serviços de 
saúde e diagnóstico precoce da patologia e maior preocupação destas com a prevenção das complicações e hábitos saudáveis do que o sexo masculino.

De acordo com Andrade et al. (2014), a predominância de mulheres em relação aos homens pode estar relacionada à maior expectativa de vida por parte da população feminina e também à resistência masculina em participação de grupos que promovam a saúde. A motivação feminina em participar das atividades de lazer é diferente da masculina, pois as mulheres, mais que os homens, buscam entrar em contato com novas pessoas e ampliar o círculo de amizades. Além disso, as mulheres têm maior atenção com a saúde e com o autocuidado.

Em relação à avaliação do estado nutricional, sob os distintos métodos: índice de massa corporal (IMC), perímetro da cintura (PC), perímetro da panturrilha $(\mathrm{PP})$, perímetro do braço $(\mathrm{PB})$, perímetro muscular do braço (PMB), dobra cutânea tricipital (DCT) e miniavaliação nutricional (MAN), descritos na Tabela 2, observamos, frequência de sobrepeso (52,2\%) nos idosos estudados, segundo o IMC, o sexo feminino apresentou $59,4 \%$ e o sexo masculino $35,8 \%$. Risco muito elevado $(69,6 \%)$ para o desenvolvimento de doenças cardiovasculares (87,5\% no sexo feminino e $28,6 \%$ no sexo masculino), conforme a PC. Em relação à $\mathrm{PP}, 93,5 \%$ dos idosos não apresentam depleção muscular (no sexo masculino $100 \%$ e no sexo feminino $90,6 \%$. Quanto à CB, verificou-se que $63 \%$ dos idosos apresentaram eutrofia $(59,4 \%$ no sexo feminino e $71,5 \%$ no sexo masculino), o que foi semelhante à $\mathrm{PMB}$, que teve frequência de eutrofia de $69,6 \%$ ( $78,1 \%$ no sexo feminino e $50 \%$ no sexo masculino). A DCT apresentou frequência de obesidade de $45,7 \%$ ( $85,8 \%$ no sexo masculino e $28,1 \%$ no sexo feminino). Ao avaliar o estado nutricional através da MAN, nota-se que $89,1 \%$ dos idosos não apresentam risco nutricional ( $87,5 \%$ no sexo feminino e $92,9 \%$ no sexo masculino). 
0
0
0
$\square$
$\square$
$\square$

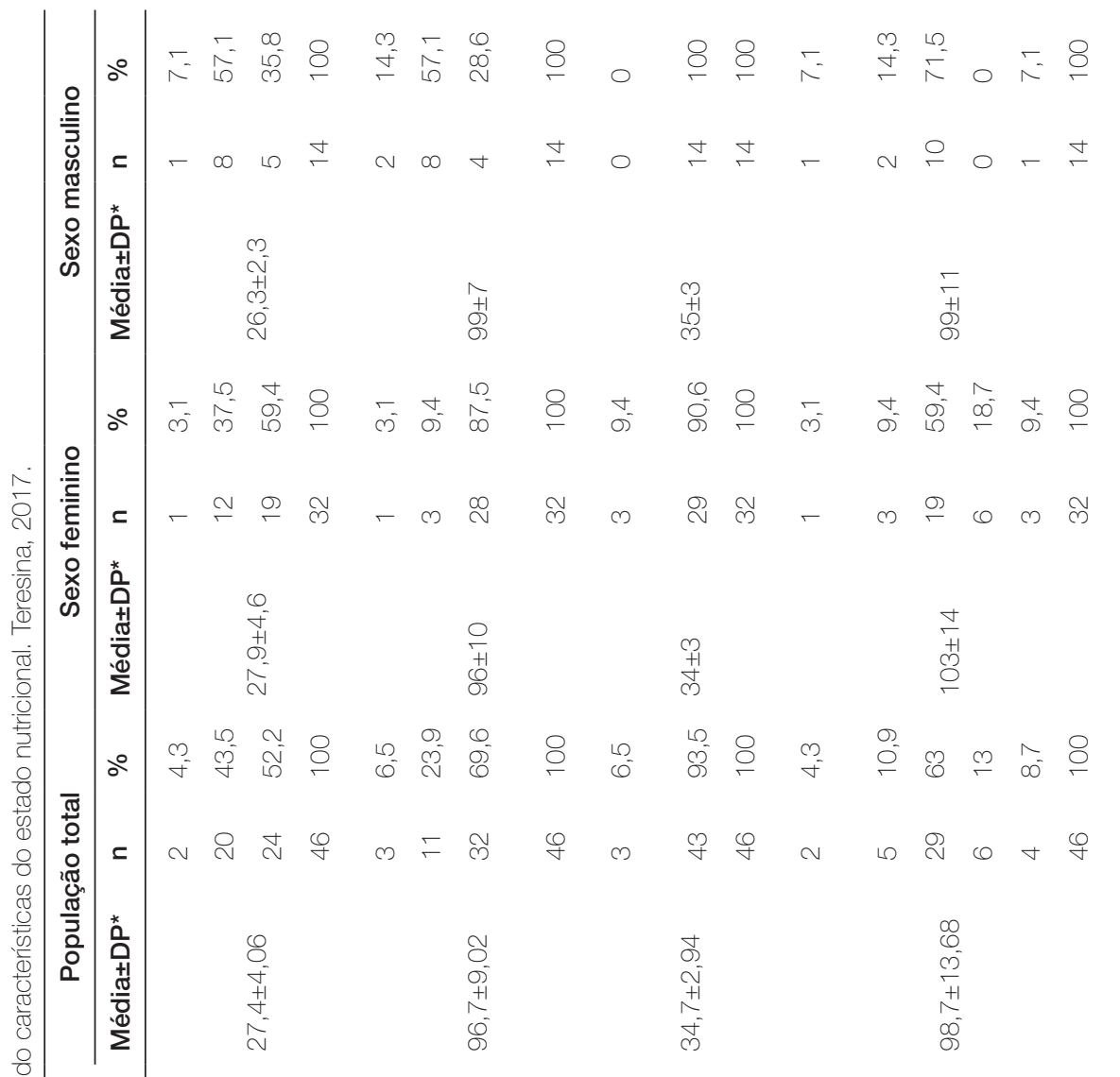

i

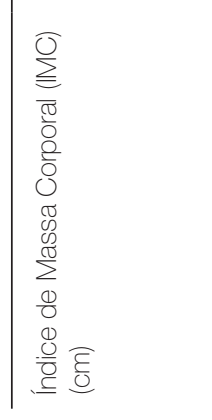

$\frac{E}{0}$
0
0
0
$\frac{0}{5}$
$\frac{0}{0}$
$\frac{\pi}{0}$
0
0
$\frac{0}{0}$
$\frac{0}{0}$
0

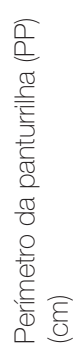

$\frac{\varepsilon}{0}$
0
0
0
0
0
$\frac{\pi}{0}$
0
0
0
0
0
0
$\frac{0}{0}$
0 


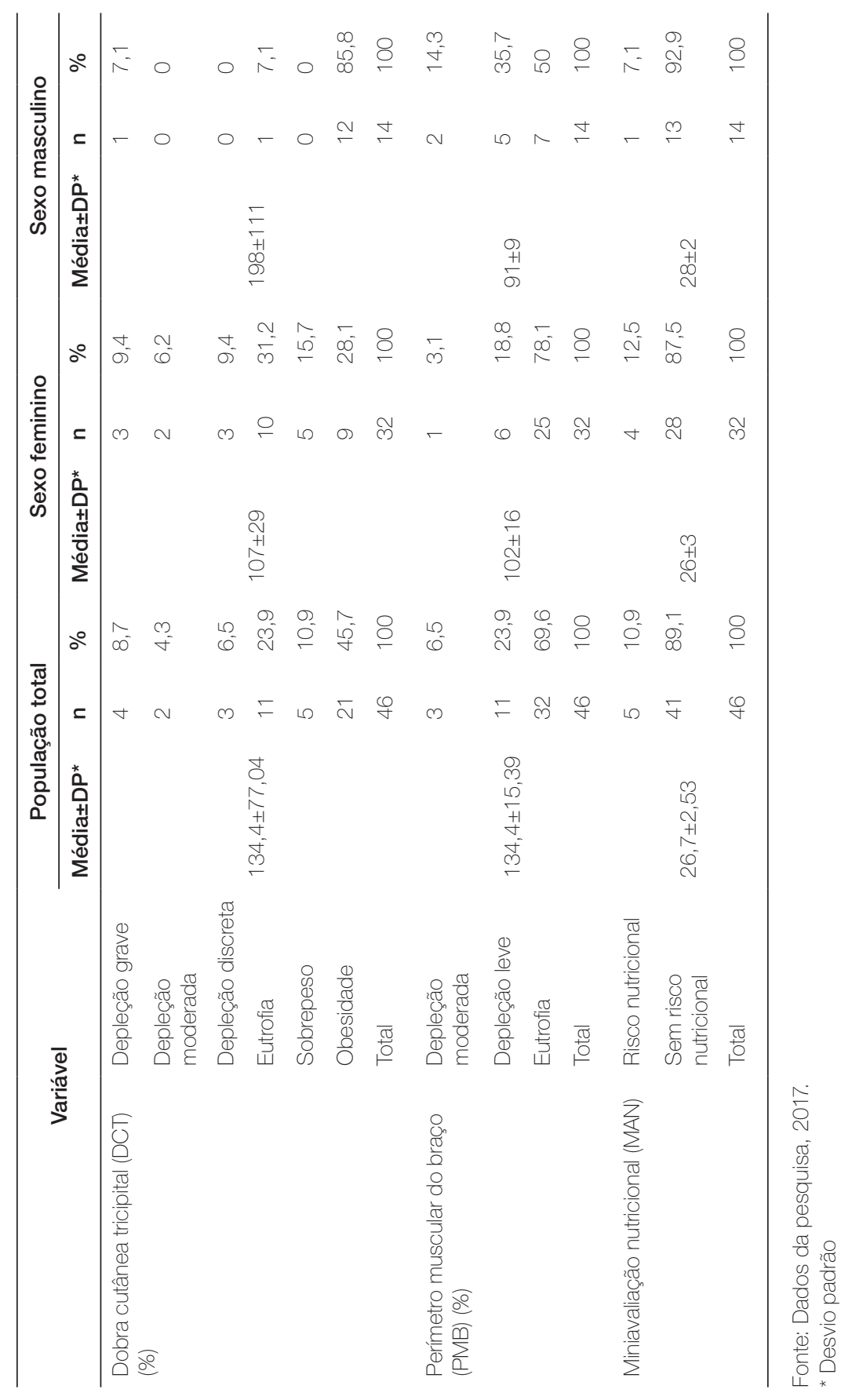

0
0
0
$\leftarrow$
$\square$
$\varangle$

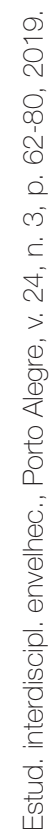


A significativa taxa de sobrepeso encontrada merece atenção especial, uma vez que aumenta as chances de complicações decorrentes do diabetes mellitus. Assim, são importantes as ações de educação nutricional, bem como o incentivo à prática de educação física como forma de prevenção e diminuição do sobrepeso e obesidade. Segundo a Sociedade Brasileira de Diabetes (2016), uma redução de 5 a 10\% do peso corporal no início do tratamento pode melhorar substancialmente a sensibilidade à insulina, característica da patologia, além do controle glicêmico, hipertensão, triglicerídeos e colesterol dos portadores DM2.

No estudo de Pereira et al. (2015), os idosos apresentaram prevalência de sobrepeso (60\%), segundo o IMC, resultado idêntico ao da pesquisa de Garcia, Fischer e Poll (2016), que indica que, na população estudada, 60\% dos idosos diabéticos estavam com sobrepeso, corroborando com o presente estudo.

A PC avalia o risco de desenvolvimento de doenças cardiovasculares (DCVS) e patologias associadas à obesidade. Na pesquisa de Freitas et al. (2017), a maioria dos idosos apresentou risco muito elevado (69,9\%), resultados semelhantes aos do presente estudo. Na pesquisa realizada por Christmann et al. (2013), constatou-se que $82,9 \%$ das idosas apresentaram risco muito elevado para DCVS e os idosos 17,1\%. Cabe destacar ainda que, na pesquisa de Magalhães, Ibiapina e Carvalho (2014) com idosos residentes em Teresina (PI), o sexo feminino apresentou risco muito elevado $(42,56 \%)$ e o sexo masculino $5,4 \%$, dados semelhantes ao presente estudo.

Segundo Pereira, Spyrides e Andrade (2016), o envelhecimento é acompanhado por diversas alterações fisiológicas, dentre as quais estão as mudanças na composição corporal que são decorrentes do aumento de massa gorda e diminuição da massa magra. Entretanto, nesse processo, não ocorre só o aumento da deposição de gordura, mas também a redistribuição desta. Além disso, os idosos da presente pesquisa são portadores de diabetes mellitus tipo 2, de acordo com a literatura em decorrência da resistência à insulina, um dos sintomas é o ganho de peso, pois, a hiperinsulinemia não tratada impulsiona o ganho de tecido adiposo.

No estudo de Moreira et al. (2013), realizado em Ceilândia (DF), 90\% dos idosos diabéticos estudados apresentaram valores adequados no PP, conforme a classificação, corroborando com a presente pesquisa, assim como, no trabalho realizado por Medeiros et al. (2017), em Recife (PE), a PP apresentou média acima de $31 \mathrm{~cm}$ para ambos os sexos, ou seja, não demonstraram depleção muscular.

A PB e a PMB apresentaram frequência de eutrofia. Na pesquisa de Santos e Saroh (2009), os valores de eutrofia (37,50\%) da PB foram menores 
e o sobrepeso $(34,38 \%)$ foi prevalente. Na avaliação da PMB, os indivíduos estudados apresentaram depleção muscular de $71,43 \%$, resultados divergentes aos do presente estudo.

A PCT apresentou frequência de obesidade nos idosos avaliados, este resultado deve-se ao elevado percentual de tecido adiposo encontrado nesta região, fato preocupante devido às complicações decorrentes desta patologia. A pesquisa de Silva et al. (2015), realizada com idosos atendidos em um ambulatório de Niterói (RJ), indicou que ambos os sexos estavam eutróficos, de acordo com a DCT. No estudo de Menezes, Souza e Mauricce (2008), realizado com idosos não diabéticos em Fortaleza (CE), os valores da DCT mostraram que $54,4 \%$ destes foram considerados eutróficos, diferentemente dos resultados da presente pesquisa. A literatura estudada informa que diabéticos apresentam maiores taxas de sobrepeso e obesidade quando comparados a indivíduos sadios.

De acordo com Santos et al. (2013), a obesidade é responsável por processos fisiopatológicos no indivíduo, como resistência à insulina, alterações hormonais, inflamação crônica, entre outros, causando complicações que comprometem a QV do portador e até mesmo a morte prematura. O excesso de peso em idosos contribui para o desenvolvimento de patologias, como a obesidade sarcopênica, na qual há diminuição da massa muscular, reduzindo o número e tamanho das fibras musculares, assim como a síntese de proteína e função mitocondrial. Conforme Pelegrini et al. (2011), a obesidade aumenta a mortalidade por outras patologias, tais como: doenças cardiovasculares, dislipidemias, doenças ósteo-articulares, doenças hepáticas e biliares, diabetes mellitus e certos tipos de cânceres.

Ao avaliar o estado nutricional através da MAN, os idosos não apresentam risco nutricional. Na pesquisa de Santos et al. (2017), 90\% dos idosos diabéticos não apresentaram risco nutricional. Da mesma forma, o estudo de Cavalcante, Coutinho e Burgos (2017), realizado em Recife (PE), indica que os idosos diabéticos avaliados não apresentaram risco nutricional $(91,6 \%)$, resultados equivalentes aos da presente pesquisa.

Na Tabela 3 estão descritos os escores (médias) dos domínios que compõem o questionário SF-36 utilizado para avaliar a qualidade de vida. 
Tabela 3 - Resultados do questionário de qualidade de vida SF-36. Teresina, 2017.

\begin{tabular}{|c|c|c|c|c|c|c|}
\hline \multirow{2}{*}{ Domínio } & \multicolumn{2}{|c|}{ População total } & \multicolumn{2}{|c|}{ Sexo feminino } & \multicolumn{2}{|c|}{ Sexo masculino } \\
\hline & $\mathrm{n}$ & Média $\pm D P^{*}$ & $\mathrm{n}$ & Média $\pm D P^{*}$ & $\mathrm{n}$ & Média $\pm D P^{*}$ \\
\hline Capacidade funcional & 46 & $76,10 \pm 27,7$ & 32 & $73,53 \pm 27,53$ & 14 & $84,28 \pm 27,45$ \\
\hline $\begin{array}{l}\text { Limitação por } \\
\text { aspectos físicos }\end{array}$ & 46 & $72,28 \pm 40,5$ & 32 & $68,96 \pm 42,75$ & 14 & $82,14 \pm 37,25$ \\
\hline Dor & 46 & $65,63 \pm 24,3$ & 32 & $58,31 \pm 24,07$ & 14 & $82,36 \pm 15,19$ \\
\hline Estado geral da saúde & 46 & $59,67 \pm 20,7$ & 32 & $57,97 \pm 21,94$ & 14 & $66,86 \pm 20,62$ \\
\hline Vitalidade & 46 & $75,32 \pm 18,2$ & 32 & $75,68 \pm 17,08$ & 14 & $77,78 \pm 21,36$ \\
\hline Aspectos sociais & 46 & $88,43 \pm 19,1$ & 32 & $86,06 \pm 21,39$ & 14 & $94,86 \pm 12,61$ \\
\hline Aspectos emocionais & 46 & $84,76 \pm 31,2$ & 32 & $84,34 \pm 31,72$ & 14 & $85,71 \pm 31,28$ \\
\hline Saúde mental & 46 & $81,28 \pm 16,6$ & 32 & $79,09 \pm 16,11$ & 14 & $86,28 \pm 17,24$ \\
\hline Total do SF-36 & 46 & $75,43 \pm 8,97$ & 32 & $72,99 \pm 10,01$ & 14 & $82,53 \pm 7,48$ \\
\hline
\end{tabular}

Fonte: Dados da pesquisa, 2017.

* Desvio padrão.

Os resultados da Tabela 3 revelam que o domínio aspectos sociais $(88,43)$ apresentou a maior média, seguido pelo domínio aspectos emocionais $(84,76)$. As menores médias foram do domínio estado geral da saúde $(59,67)$ e dor $(65,63)$ na população em geral. Os resultados indicam que os idosos pesquisados possuem qualidade de vida, uma vez que apresentaram em seis domínios (capacidade funcional, limitação por aspectos físicos, vitalidade, aspectos sociais, aspectos emocionais e saúde mental) médias próximas a 100 (zero corresponde a uma pior qualidade de vida e 100 a uma melhor qualidade de vida) e média total dos domínios de 75,43 na população total, 72,99 no sexo feminino e 82,53 no sexo masculino, indicando boa qualidade de vida, conforme o questionário SF-36.

No estudo de Neves et al. (2018), realizado em Diamantino (RS), com idosos diabéticos, as menores médias foram nos domínios estado geral da saúde $(61,6)$ e dor $(52,7)$, corroborando com a presente pesquisa. Entretanto, a média do SF-36 foi menor, 66,4. Na pesquisa de Sousa et al. (2016), com idosos diabéticos em Cajazeiras (PB), a análise de domínios do SF-36 apresentou menores médias nos domínios dor (26,76), estado geral da saúde $(40,81)$, aspectos sociais $(42,46)$ e maior média no domínio saúde mental $(74,76)$, pontuações menores ao presente estudo.

Segundo Benfica e Rabelo (2011), envelhecimento é um período de alta incidência de patologias, na maioria das vezes, estas são acompanhadas de dor 
que podem interferir na $\mathrm{QV}$ do idoso. A dor altera a rotina do idoso e limita algumas de suas decisões, além de afetar o psicológico deste, influenciando no seu estado geral de saúde, pois reflete na percepção e expectativas do indivíduo em relação à sua saúde e como este se sente no seu dia a dia.

De acordo com Celich e Galon (2009), as dores dos idosos são atribuídas à idade e consideradas próprias ao processo de envelhecimento. Estas devem ser investigadas e avaliadas por profissionais de saúde para o tratamento específico, como forma de melhorar a $\mathrm{QV}$, uma vez que influenciam negativamente na rotina dos idosos.

No estudo de Souza et al. (2016), realizado com 50 idosos diabéticos do interior da Bahia, a pior pontuação encontrada foi no domínio psicológico (51,30\%) e a melhor pontuação no domínio relações sociais $(79,92)$. Diferentemente, a pesquisa de Freitas et al. (2017) com idosos não diabéticos, indica maior média no domínio psicológico $(83,33)$, seguido pelo domínio social $(75,42)$. Os idosos que possuem uma vida socialmente ativa, que vivem integrados na comunidade, possuem melhor $\mathrm{QV}$, uma vez que possuem suporte emocional e sensação de segurança que são decorrentes de um senso de pertencimento e integração.

Segundo Souza et al. (2016), o domínio de relações sociais envolve as relações pessoais, o suporte social. Estes contribuem na QV dos idosos, pois as pessoas que possuem bons relacionamentos nos meios sociais e não se isolam, apresentam maior satisfação pessoal, o que, sem dúvida, minimiza a ocorrência de possíveis sentimentos de tristeza. Conforme Pereira, Alvarez e Traebert (2011), o domínio psicológico aborda questões relacionadas aos sentimentos, espiritualidade, religião e autoestima do indivíduo. Além da saúde mental, como alterações comportamentais, distúrbios de ansiedade e depressão, entre outros problemas emocionais.

Na pesquisa de Santos et al. (2013), realizada com 104 idosos diabéticos residentes da zona urbana de Uberaba, o menor escore de QV relacionou-se ao domínio físico $(54,16)$ e o maior escore às relações sociais $(67,56)$, resultado semelhante ao da presente pesquisa. Isso indica que os idosos possuem bons relacionamentos com a comunidade e satisfação social.

Conforme Girardi et al. (2015), o aspecto físico avalia a limitação da saúde devido a problemas físicos, redução de atividades e dificuldade de realizar tarefas, e o domínio relações sociais, analisa o relacionamento do indivíduo com a família, amigos, grupos e sua integração na sociedade.

Conforme os resultados do questionário SF-36, nota-se que a QV deste público é influenciada por dois domínios, dor e estado geral da saúde, pois o envelhecimento é caracterizado por alterações fisiológicas no organismo e surgimento de doenças, e estas podem ocasionar dor e o DM modifica os hábitos 
de vida dos portadores, alterando o estado geral de saúde destes. Entretanto, a média total do SF-36 indica QV nestes idosos, pois esta engloba uma série de fatores como: bem-estar, estado emocional, estilo de vida, aspectos físicos, satisfação pessoal, além da dor e percepção da saúde. Os idosos avaliados são participantes de uma associação para diabéticos, permitindo a estes uma integração social com outros diabéticos e conhecimentos sobre o controle e prevenção das complicações decorrentes do DM, favorecendo processo de envelhecimento saudável e melhoria da QV.

\section{Conclusão}

Através desta pesquisa foi possível verificar que os idosos com diabetes tipo 2 possuem elevado percentual de sobrepeso e obesidade, conforme o índice de massa corporal e adequação da dobra cutânea tricipital, entretanto, apresentam boa qualidade de vida, apesar de verificadas inadequações no estado nutricional, uma vez que apresentaram nos domínios capacidade funcional, limitação por aspectos físicos, vitalidade, aspectos sociais, aspectos emocionais, saúde mental e média total próximos a cem. Constata-se, assim, a importância de implementações de ações de educação nutricional dentro das políticas públicas de promoção da saúde, como forma de assegurar qualidade de vida. No entanto, destaca-se a necessidade de novos estudos com maiores populações a fim de ampliar o conjunto de evidências sobre o estado nutricional e qualidade de vida desta população.

\section{EVALUATION OF THE NUTRITIONAL STATUS AND QUALITY OF LIFE OF ELDERLY PATIENTS WITH DIABETES MELLITUS TYPE 2}

abstract

The objective of the research was to analyze the nutritional status and quality of life of elderly patients with diabetes mellitus type 2. The population was composed of 46 volunteers that also were carriers of type 2 diabetes, with age range between 60 and 85 years of age, all members of the diabetic association located in Teresina (Piauí). Older adults were subjected to a demographic form and then the evaluation of nutritional status was performed through nutritional small evaluation and anthropometric data. Finally, the quality of life 
was evaluated through the Short Form-36 questionnaire. According to the sociodemographic variables, it was found that most of the participants are female (69.6\%), aged between 60 and 65 years of age (54.3\%), married (63\%), graduated from middle school (54.3\%) and income of 1 (39.5\%) and 2-3 minimum wages (39.1\%). In the evaluation of the nutritional state, according to the body mass index, predominated it overweight in 52.2\%; Through evaluation of triceps cutis, obesity was found in $45.7 \%$ of the participants; according to the waist circumference, there was a very high risk for the development of cardiovascular diseases in 69.65\% of subjects researched; it was also found that $88.43 \%$ of older adults did not have a nutritional risk when assessed through nutritional small evaluation. As for the quality of life analysis, the smallest averages (scores) were found in the general state of Health (59.67\%) and Pain (65.63\%). Consequently, older adults patients with type 2 diabetes evaluated have changes in the nutritional state; however they present good quality life.

keywords

Diabetes Mellitus. Nutritional Status. Quality of Life. Elderly.

referências

ANDRADE, Ankilma do Nascimento et al. Percepção de idosos sobre grupo de convivência: estudo na cidade de Cajazeiras (PB). Revista Brasileira de Geriatria e Gerontologia, Rio de Janeiro, v. 17, n. 1, p. 39-48, 2014. Disponível em: http://www.redalyc. org/articulo.oa?id=403838834005. Acesso em: 19 nov. 2017.

BENFICA, Marema Pereira; RABELO, Dóris Firmino. O idoso com diabetes mellitus: fatores psicológicos, comportamentais e sociais. Perquirere: Revista do Centro Universitário de Patos de Minas, MG, v. 1, n. 8, ago. 2011. Disponível em: https://revistas.unipam. edu.br/index.php/perquirere/issue/view/95. Acesso em: 19 nov. 2017.

BLACKBURN, George; THORNTON, Paul. Nutritional assessment of the hospitalized patients. Medical Clinics North America, New York, v. 63, p. 1103-1115, 1979.

CAVALCANTE, Lilian Souza; COUTINHO, Paloma Travassos de Queiróz; BURGOS, Maria Goretti Pessoa de Araújo. Aplicabilidade da MAN: Mini Avaliação Nutricional em idosos diabéticos. Nutrición clínica y Dietética Hospitalar, Madrid, v. 37, n. 1, p. 67-74, 2017. Disponível em: http://revista.nutricion.org/PDF/iliansouza.pdf. Acesso em: 25 mar. 2018.

CELICH, Kátia Lilian Sedrez; GALON, Cátia. Dor crônica em idosos e sua influência nas atividades da vida diária e convivência social. Revista Brasileira de Geriatria e Gerontologia, Rio de Janeiro, v. 12, n. 3, p. 345-359, 2009. Disponível em: http://www. scielo.br/pdf/rbgg/v12n3/1981-2256-rbgg-12-03-00345.pdf. Acesso em: 19 nov. 2017.

CHRISTMANN, Aline Cristina et al. Perfil de Risco de Doenças Cardiovasculares e Estado Nutricional de ldosos Ativos de Guarapuava, Paraná. UNOPAR Científica: Ciências Biológicas e da Saúde, Londrina, v. 15, p. 349-356, 2013. Disponível em: http://www.pgsskroton.com. br/seer/index.php/JHealthSci/article/view/570/538. Acesso em: 15 mar. 2018. 
CICONELLI, Rozana Mesquita. Tradução para o português e validação do questionário genérico de avaliação de qualidade de vida Medical Outcomes Study 36-Item Short-Form Health Survey (SF-36). Tese (Doutorado em Medicina) - Escola Paulista de Medicina, Universidade Federal de São Paulo, São Paulo, 1997. Disponível em: http://repositorio. unifesp.br/handle/11600/15360. Acesso em: 20 ago. 2017.

COQUEIRO, Raildo da Silva et al. Medidas auto-referidas são válidas para avaliação do estado nutricional. Revista Brasileira de Cineantropometria e Desempenho Humano, Florianópolis, v. 11, n. 1, p. 113-119, 2009. Disponível em: http://www.rbone.com.br/ index.php/rbone/article/view/274/254. Acesso em: 2 jul. 2019.

DEUS, Karine Junqueira; CONCEIÇÃO, Raquel Santos. Diabetes mellitus tipo 2: a relação genética-nutrientes. Nutrire: Revista Brasileira da Sociedade Brasileira de Alimentação e Nutrição, São Paulo, v. 37, n. 2, p. 199-214, ago. 2012. Disponível em: http://files.bvs. br/upload/S/1519-8928/2012/v37n2/a3251.pdf. Acesso em: 26 mar. 2018.

DIAZ, Naiana et al. O impacto do diabetes mellitus tipo 2 na qualidade de vida. Revista Médica da UFPR, Curitiba, v. 3, n. 1, p. 5-12, 2016. Disponível em: http://revistas.ufpr. br/revmedicaufpr/article/view/46380/pdf. Acesso em: 2 jul. 2019.

ESTEVES, Marlene et al. Qualidade de vida de idosos hipertensos e diabéticos em um serviço ambulatorial. Revista de Medicina de Ribeirão Preto e do Hospital das Clínicas da FMRP, Ribeirão Preto, SP, v. 50, n. 1, p. 18-28, 2017. Disponível em: https://idonline. emnuvens.com.br/id/article/view/599/856. Acesso em: 15 mar. 2018.

FARIA, Heloisa Turcatto Gimenes et al. Qualidade de vida de pacientes com diabetes mellitus antes e após participação em programa educativo. Revista da Escola de. Enfermagem da USP, São Paulo, v. 47, n. 2, p. 348-354, 2013. Disponível em: http://www.scielo.br/scielo. php?script=sci_arttext\&pid=S0080-62342013000200011. Acesso em: 19 mar. 2017.

FENG, Xiaoqi; ASTELL-BURT, Thomas. Impact of a type 2 diabetes diagnosis on mental health, quality of life, and social contacts: a longitudinal study. BMJ Open Diabetes Research and Care, Londres, 2017. Disponivel em: https://www.ncbi.nlm.nih.gov/ pubmed/28243446. Acesso em: 17 mar. 2018.

FREITAS, Ana Paula et al. Relação da qualidade de vida com o estado nutricional de idosos. Revista Brasileira de Qualidade de Vida, Ponta Grossa, v. 9, n. 1, p. 30-44, jan./ mar. 2017. Disponível em: https://periodicos.utfpr.edu.br/rbqv/article/viewFile/5236/3583. Acesso em: 19 nov. 2017.

GARCIA, Carina; FISCHER, Maiara de Queiroz; POLL, Fabiana Assmann. Estado nutricional e as comorbidades associadas ao diabetes mellitus tipo 2 no idoso. Revista Estudos interdisciplinares sobre o envelhecimento, Porto Alegre, v. 21, n. 1, p. 205-216, 2016. Disponível em: http://www.seer.ufrgs.br/RevEnvelhecer/article/view/59049. Acesso em: 19 nov. 2017

GIRARDI, Camila Ely et al. Qualidade de vida de pessoas em grupos de convivência com diabetes mellitus tipo 2. Revista de Enfermagem UFPE, Recife, v. 9, n. 4, p. 7239-7246, abr. 2015. Disponível em: http://www.revista.ufpe.br/revistaenfermagem/index. php/revista/article/viewArticle/6615. Acesso em: 19 nov. 2017.

GUIGOZ, Yves. The Mini-Nutritional Assessment (MNA®) review of the literature: what does it tell us? J. Nutr. Health Aging, New York, v. 10, p. 466-487, 2006

LIPSCHITZ, David. Screening for nutricional status in the elderly. PrimareCare, Philadelphia, Saunders, v. 21 n. 1, p. 55-57, 1994.

MAGALHÃES, Beatriz de Sousa; IBIAPINA, Daniela Fortes; CARVALHO, Danyella Rodrigues. Avaliação nutricional e prevalência de diabetes e hipertensão em idosos. Revista Interdisciplinar, Teresina, v. 7, n. 4, p. 131-138, 2014. Disponível em: https://revistainterdisciplinar.uninovafapi.edu.br/index.php/revinter/article/view/476. Acesso em: 9 maio 2018.

MEDEIROS, Gabriela Magalhães et al. Efeitos do acompanhamento nutricional sobre os parâmetros antropométricos em idosos diabéticos a nível ambulatorial. Revista Nutrición clínica y Dietética Hospitalar, Madrid, v. 37, n. 3, p. 29-34, 2017. Disponível em: http:// revista.nutricion.org/PDF/GMedeiros.pdf. Acesso em: 19 nov. 2017. 
MENEZES, Tarciana Nobre; SOUZA, José Maria Pacheco; MARUCCI, Maria de Fátima Nunes. Avaliação do estado nutricional dos idosos residentes em Fortaleza/CE: o uso de diferentes indicadores antropométricos. Revista Brasileira de Cineantropometria e Desempenho Humano, Florianópolis, v. 10, n. 4, p. 315-322, 2008. Disponível em: http://www.producao.usp.br/handle/BDPI/14197. Acesso em: 20 nov. 2017.

MOREIRA, Sthefânya Shabryny Cavalcante Regis. Avaliação do estado nutricional de idosos portadores de diabetes tipo 2 acompanhados no Centro de Saúde. Monografia (Bacharelado em Enfermagem) - Faculdade de Ceilândia da Universidade de Brasília, Ceilândia, 2013. Disponível em: http://bdm.unb.br/bitstream/10483/6985/1/2013_SthefanyaShabrynyCavalcanteRegisMoreira.pdf. Acesso em: 19 nov. 2017.

NATIONAL INSTITUTES OF HEALTH (NIH). The practical guide identification, evaluation, and treatment of overweight and obesity in adults. Bethesda, MD: National Institutes of Health, 2000. Publication n. 00-4084.

NEVES, Tiago et al. Qualidade de vida de idosos diabéticos tipo 2 e não diabéticos. Revista brasileira de qualidade de vida, Ponta Grossa, v. 10, n. 3, jul./set. 2018. Disponível em: http://www.scielo.br/pdf/rbgg/v21n2/pt_1809-9823-rbgg-21-02-00176.pdf. Acesso em: 2 jul. 2019.

OLIVEIRA, Beatriz Campos et al. Avaliação da qualidade de vida em idosos da comunidade. Revista Brasileira em Promoção da Saúde, Fortaleza, v. 30, n. 3, p. 1-10, jul./ set. 2017. Disponive/ em: http://periodicos.unifor.br/RBPS/article/view/5879. Acesso em: 15 mar. 2018.

PALMEIRA, Catia Suely; PINTO, Sayonara Rocha. Perfil epidemiológico de pacientes com diabetes mellitus em Salvador, Bahia, Brasil (2002-2012). Revista Baiana de Enfermagem, Salvador, v. 29, n. 3, p. 240-249, jul./set. 2015. Disponível em: https://portalseer. ufba.br/index.php/enfermagem/article/view/13158/pdf_7. Acesso em: 21 mar. 2018.

PELEGRINI, Andreia et al. Diabetes mellitus auto-referido e sua associação com excesso de peso em idosos. Revista Brasileira de Cineantropometria e Desempenho Humano, Florianópolis, v. 13, n. 6, p. 442-447, 2011. Disponível em: http://www.scielo.br/pdf/ rbcdh/v13n6/a06v13n6.pdf. Acesso em: 19 nov. 2017.

PEREIRA, Ingrid Freitas da Silva; SPYRIDES, Maria Helena Constantino; ANDRADE, Lára de Melo Barbosa. Estado nutricional de idosos no Brasil: uma abordagem multinível. Cadernos de Saúde Pública, Rio de Janeiro, v. 32, n. 5, maio 2016. Disponível em: http://www.scielo.br/scielo.php?pid=S0102-311X2016000500709\&script=sci_abstract\&tlng=pt. Acesso em: 20 nov. 2017.

PEREIRA, Keila Cristina Rausch; ALVAREZ, Angela Maria; TRAEBERT, Jefferson Luiz. Contribuição das condições sociodemográficas para a percepção da qualidade de vida em idosos. Revista Brasileira de Geriatria e Gerontologia, Rio de Janeiro, v. 14 n. 1, p. 85-95, 2011. Disponível em: http://www.scielo.br/pdf/rbgg/v14n1/a10v14n1. pdf. Acesso em: 15 mar. 2018.

PEREIRA, Laianny Krizia Maia et al. Estado nutricional de idosos, portadores de diabetes mellitus tipo 2 atendidos em uma unidade básica de saúde, Água Branca/PB. Anais CIEH, Campina Grande, v. 2, n. 1, p. 1-10, 2015. Disponivel em: http://www.editorarealize.com. br/revistas/cieh/trabalhos/TRABALHO_EVO4O_MD2_SA7_ID2103_28072015120242. pdf. Acesso em: 19 nov. 2017.

SANTOS, Érica Aparecida et al. Morbidades e qualidade de vida de idosos com diabetes mellitus residentes nas zonas rural e urbana. Revista da Escola de Enfermagem da USP, São Paulo, v. 47, n. 2, p. 393-400, 2013. Disponível em: http://www.scielo.br/ pdf/reeusp/v47n2/17.pdf. Acesso em: 19 nov. 2017.

SANTOS, Leila Martins et al. Avaliação do hábito alimentar e estado nutricional de idosos com diabetes mellitus tipo 2 atendidos na atenção básica de saúde do município de Porteiras/ CE. Revista E-ciência, Juazeiro do Norte, v. 5, n. 1, p. 69-77, 2017. Disponível em: http:// www.revistafin.com.br/revista/index.php/eciencia/article/view/271. Acesso em: 19 nov. 2017. 
SANTOS, Xênia Pimenta da Silva; SARON, Margareth Lopes Galvão. Perfil nutricional de idosos, portadores de diabetes mellitus tipo 2 atendidos pelo programa saúde da família. ENCONTRO LATINO AMERICANO DE INICIAÇÃO CIENTÍFICA, 13.; ENCONTRO LATINO AMERICANO DE PÓS-GRADUAÇÃO, 9., 2009, São José dos Campos. Anais: Perfil nutricional de idosos, portadores de diabetes mellitus tipo 2 atendidos pelo programa saúde da família. São José dos Campos: Universidade do Vale do Paraíba, 2009. p. 1-6. Disponível em: http://www.inicepg.univap.br/cd/INIC_2009/anais/arquivos/ RE_0275_0469_01.pdf. Acesso em: 19 nov. 2017.

SILVA, Ana Luiza Sant'Anna da Costa et al. Avaliação antropométrica de idosos atendidos no Ambulatório de Nutrição do Centro de Referência em Assistência à Saúde do Idoso da Universidade Federal Fluminense, no município de Niterói/RJ. Revista Demetra: Alimentação, Nutrição \& Saúde, v. 10, n. 2. p. 361-374, 2015. Disponível em: http://www.e-publicacoes.uerj.br/index.php/demetra/article/view/15949. Acesso em: 9 maio 2018.

SOCIEDADE BRASILEIRA DEDIABETES. Diretrizes da Sociedade Brasileira de Diabetes: 2015-2016. São Paulo: AC Farmacêutica, 2016. Disponível em: http://www. diabetes.org. br/profissionais/images/docs/DIRETRIZES-SBD-2015-2016.pdf. Acesso em: 18 mar. 2018.

SOUSA, Eliane Leite et al. Qualidade de vida e fatores associados à saúde de idosos diabéticos. Revista Enfermagem UERJ, Rio de Janeiro, v. 24, n. 5, 2016. Disponível em: http://www.facenf.uerj.br/v24n5/v24n5a02.pdf. Acesso em: 2 jul. 2019.

SOUZA, Danilo Pereira et al. Qualidade de Vida em Idosos portadores de Hipertensão Arterial e Diabetes. Rev. Id on Line: Revista Multiprofissional e de Psicologia, Jaboatão dos Guararapes, PE, v. 10, n. 31, out./nov. 2016. Disponível em: https://idonline.emnuvens.com.br/id/article/view/599/856. Acesso em: 15 mar. 2018.

SOUZA, Márcia Ferreira Candido; ARAÚJO, Viviane Ferreira. Adequação do consumo e evolução antropométrica após educação nutricional de pacientes com diabetes mellitus tipo 2. Revista Demetra: alimentação, nutrição e saúde, Rio de Janeiro, v. 10, n. 1, p. 159-172, 2015. Disponível em: http://www.e-publicacoes.uerj.br/index.php/ demetra/article/viewFile/13376/12222. Acesso em: 18 mar. 2018.

TAVARES, Darlene Mara dos Santos; RODRIGUES, Rosalina Aparecida Partezani. Indicadores sociodemográficos e de saúde de idosos portadores e não portadores de diabetes. Revista Eletrônica de Enfermagem, Goiânia, v. 10, n. 4, p. 906-914, 2008. Disponível em: https://www.fen.ufg.br/fen_revista/v10/n4/pdf/v10n4a03.pdf. Acesso em: 19 nov. 2017.

UMBELINO, Adriana Ferreira; SERRANO, Hiara Miguel Stanciola; CRUZ, Nilcemar Rodrigues. Avaliação nutricional e clínica em pacientes diabéticos hospitalizados. Nutrir Gerais: Revista Digital de Nutrição, Ipatinga, v. 2, n. 2. fev./jul. 2008. Disponível em: https://www.unilestemg. br/nutrirgerais/downloads/artigos/avaliacao_nutricional.pdf. Acesso em: 15 mar. 2018.

VIEGAS-PEREIRA, Ana Paula Franco; RODRIGUES, Roberto Nascimento; MACHADO, Carla Jorge. Fatores associados à prevalência de diabetes auto-referido entre idosos de Minas Gerais. Revista brasileira de Estudos de Populações, São Paulo, v. 25, n. 2, p. 365-376, jul./dez. 2008. Disponível em: http://www.scielo.br/pdf/rbepop/v25n2/ v25n2a11.pdf. Acesso em: 19 nov. 2017.

ZULIAN, Luana Rosas et al. Qualidade de vida de pacientes com diabetes utilizando o instrumento Diabetes 39 (D-39). Revista Gaúcha de Enfermagem, Porto Alegre, v. 3, n. 34, p. 138-146, 2013. Disponível em: http://www.scielo.br/scielo.php?script=sci_arttext\&pid=S1983-14472013000300018. Acesso em: 1 abr. 2018

Data de Submissão: 02/08/2018

Data de Aprovação: 18/09/2019 